\title{
THE ALHAMBRA SURVEY: EVOLUTION OF GALAXY SPECTRAL SEGREGATION
}

\author{
Ll. Hurtado-Gil ${ }^{1,2}$, P. Arnalte-Mur ${ }^{1}$, V. J. Martínez ${ }^{1,3,4}$, A. Fernández-Soto ${ }^{2,4}$, M. Stefanon ${ }^{5}$, B. Ascaso ${ }^{6,7}$, \\ C. López-Sanjuán ${ }^{8}$, I. Márquez ${ }^{9}$, M. Pović ${ }^{9}$, K. Virronen ${ }^{8}$, J. A. L. Aguerri ${ }^{10,11}$, E. Alfaro9, T. Aparicio-Villegas ${ }^{9,12}$, \\ N. Benítez ${ }^{9}$, T. Broadhurst ${ }^{13,14}$, J. Cabrera-Caño ${ }^{15}$, F. J. Castander ${ }^{16}$, J. CePa ${ }^{10,11}$, M. Cerviño ${ }^{9,10,11}$, \\ D. Cristóbal-Hornillos ${ }^{8}$, R. M. González Delgado 9 , C. Husillos ${ }^{9}$, L. Infante ${ }^{17}$, J. Masegosa ${ }^{9}$, M. Moles ${ }^{8,9}$, \\ A. Molino ${ }^{9,18}$, A. del Olmo ${ }^{9}$, S. Paredes ${ }^{19}$, J. Perea ${ }^{9}$, F. Prada ${ }^{9,20,21}$, and J. M. Quintana ${ }^{9}$ \\ ${ }_{1}^{1}$ Observatori Astronòmic, Universitat de València, C/ Catedràtic José Beltrán 2, E-46980, Paterna, Spain \\ ${ }^{2}$ Instituto de Física de Cantabria (CSIC-UC), E-39005 Santander, Spain \\ ${ }^{3}$ Departament d'Astronomia i Astrofísica, Universitat de València, E-46100, Burjassot, Spain \\ ${ }^{4}$ Unidad Asociada Observatorio Astronómico (IFCA-UV), E-46980, Paterna, Spain \\ ${ }^{5}$ Leiden Observatory, Leiden University, P.O. Box 9513, 2300 RA Leiden, The Netherlands \\ ${ }^{6}$ GEPI, Observatoire de Paris, CNRS, Université Paris Diderot, 61, Avenue de 1Observatoire F-75014, Paris France \\ ${ }^{7}$ APC, AstroParticule et Cosmologie, Université Paris Diderot, CNRS, 10, rue Alice Domon et Léonie Duquet, 75013 Paris, France \\ ${ }^{8}$ Centro de Estudios de Física del Cosmos de Aragón, Plaza San Juan 1, E-44001 Teruel, Spain \\ ${ }^{9}$ IAA-CSIC, Glorieta de la Astronomía s/n, E-18008 Granada, Spain \\ ${ }^{10}$ Instituto de Astrofísica de Canarias, Vía Láctea s/n, E-38200 La Laguna, Tenerife, Spain \\ ${ }^{11}$ Departamento de Astrofísica, Facultad de Física, Universidad de La Laguna, E-38206 La Laguna, Spain \\ ${ }_{12}$ Observatório Nacional-MCT, Rua José Cristino, 77. CEP 20921-400, Rio de Janeiro-RJ, Brazil \\ ${ }^{13}$ Department of Theoretical Physics, University of the Basque Country UPV/EHU, E-48080 Bilbao, Spain \\ ${ }^{14}$ IKERBASQUE, Basque Foundation for Science, Bilbao, Spain \\ ${ }^{15}$ Departamento de Física Atómica, Molecular y Nuclear, Facultad de Física, Universidad de Sevilla, E-41012 Sevilla, Spain \\ ${ }^{16}$ Institut de Ciències de l'Espai (IEEC-CSIC), Facultat de Ciències, Campus UAB, E-08193 Bellaterra, Spain \\ ${ }^{17}$ Departamento de Astronomía, Pontificia Universidad Católica. 782-0436 Santiago, Chile \\ ${ }^{18}$ Instituto de Astronomía, Geofísica e Ciéncias Atmosféricas, Universidade de São Paulo, São Paulo, Brazil \\ ${ }^{19}$ Departamento de Matemática Aplicada y Estadística, Universidad Politécnica de Cartagena, C/Dr. Fleming s/n, E-30203 Cartagena, Spain \\ ${ }^{20}$ Instituto de Física Teórica, (UAM/CSIC), Universidad Autónoma de Madrid, Cantoblanco, E-28049 Madrid, Spain \\ ${ }^{21}$ Campus of International Excellence UAM+CSIC, Cantoblanco, E-28049 Madrid, Spain \\ Received 2015 June 30; accepted 2016 January 13; published 2016 February 17
}

\begin{abstract}
We study the clustering of galaxies as a function of spectral type and redshift in the range $0.35<z<1.1$ using data from the Advanced Large Homogeneous Area Medium Band Redshift Astronomical (ALHAMBRA) survey. The data cover $2.381 \mathrm{deg}^{2}$ in 7 fields, after applying a detailed angular selection mask, with accurate photometric redshifts $\left[\sigma_{z}<0.014(1+z)\right]$ down to $I_{A B}<24$. From this catalog we draw five fixed number density redshiftlimited bins. We estimate the clustering evolution for two different spectral populations selected using the ALHAMBRA-based photometric templates: quiescent and star-forming galaxies. For each sample we measure the real-space clustering using the projected correlation function. Our calculations are performed over the range [0.03, 10.0] $h^{-1} \mathrm{Mpc}$, allowing us to find a steeper trend for $r_{p} \lesssim 0.2 h^{-1} \mathrm{Mpc}$, which is especially clear for star-forming galaxies. Our analysis also shows a clear early differentiation in the clustering properties of both populations: starforming galaxies show weaker clustering with evolution in the correlation length over the analyzed redshift range, while quiescent galaxies show stronger clustering already at high redshifts and no appreciable evolution. We also perform the bias calculation where similar segregation is found, but now it is among the quiescent galaxies where a growing evolution with redshift is clearer (abrigatted). These findings clearly corroborate the well-known colordensity relation, confirming that quiescent galaxies are mainly located in dark matter halos that are more massive than those typically populated by star-forming galaxies.
\end{abstract}

Key words: cosmology: observations - galaxies: distances and redshifts - large-scale structure of universe methods: data analysis - methods: statistical

\section{INTRODUCTION}

It has been well established that different types of galaxies cluster in different ways (Davis et al. 1988; Hamilton 1988; Domínguez-Tenreiro \& Martínez 1989; Einasto 1991; Loveday et al. 1995; Guzzo et al. 1997; Li et al. 2006; Phleps et al. 2006; Martínez et al. 2010). Elliptical galaxies are preferentially located at the cores of rich galaxy clusters, i.e., in high-density environments, while spiral galaxies are the dominant population in the field (Davis \& Geller 1976; Dressler 1980; Giovanelli et al. 1986; Cucciati et al. 2006). This phenomenon, called galaxy segregation, has been confirmed in the largest galaxy redshift surveys available up to date: the $2 \mathrm{dF}$ Galaxy redshift survey (2dFGRS, Madgwick et al. 2003), the Sloan
Digital Sky Survey (SDSS, Abbas \& Sheth 2006; Zehavi et al. 2011), and the Baryonic Oscillation Spectroscopic Survey (BOSS, Guo et al. 2013). The dependence of clustering on different galaxy properties such as stellar mass, concentration index, or the strength of the $4000 \AA$ break has been studied by Li et al. (2006).

Since segregation is a consequence of the process of structure formation in the universe, it is therefore very important to understand its evolution with redshift or cosmic time. Several works have extended the analysis of segregation by color or spectral type to redshifts in the range $z \sim 0.3-1.2$ using recent spectroscopic surveys such as the VIMOS-VLT Deep Survey (VVDS, Meneux et al. 2006), the Deep 
Extragalactic Evolutionary Probe 2 survey (DEEP2, Coil et al. 2008), or the PRIsm Multi-object Survey (PRIMUS, Skibba et al. 2014). de la Torre et al. (2011), instead, used the zCOSMOS survey to study segregation by morphological type at $z \sim 0.8$. All these studies show that segregation by color or spectral type was already present at $z \sim 1$. In particular, Meneux et al. (2006) using a sample of 6500 VVDS galaxies covering half a square degree, have unambiguously established that early-type galaxies are more strongly clustered than latetype galaxies at least since redshift $z \sim 1.2$. The correlation length obtained by these authors for late-type galaxies is $r_{0} \sim 2.5 h^{-1} \mathrm{Mpc}$ at $z \sim 0.8$ and roughly twice this value for early-type galaxies. They have also calculated the relative bias between the two types of galaxies and obtained an approximately constant value $b_{\text {rel }} \sim 1.3-1.6$ for $0.2 \leqslant z \leqslant 1.2$ depending on the sample. This value is slightly larger than the one obtained by Madgwick et al. (2003) $b_{\text {rel }} \sim 1.45 \pm 0.14$ for the $2 \mathrm{dF}$ Galaxy Redshift Survey with median redshift $z=0.1$. The results obtained by Coil et al. (2008) for DEEP2 reinforced those outlined above, although the measured correlation lengths for DEEP2 galaxies are systematically slightly larger than the values reported for the VVDS sample by Meneux et al. (2006). In addition, Coil et al. (2008) have detected a significant rise of the correlation function at small scales $r_{p} \leqslant 0.2 h^{-1} \mathrm{Mpc}$ for their brighter samples. For the zCOSMOS-Bright redshift survey, de la Torre et al. (2011) also found that early-type galaxies exhibit stronger clustering than late-type galaxies on scales from 0.1 to $10 \mathrm{~h}^{-1} \mathrm{Mpc}$ already at $z \simeq 0.8$ and that the relative difference increases with cosmic time on small scales but does not significantly evolve from $z=0.8$ to $z=0$ on large scales. A similar result is reported by Skibba et al. (2014). These authors show that the clustering amplitude for the PRIMUS sample increases with color, with redder galaxies displaying stronger clustering at scales $r_{p} \leqslant 1 h^{-1} \mathrm{Mpc}$. They have also detected a color dependence within the red sequence with the reddest galaxies being more strongly correlated than their less red counterparts. This effect is absent in the blue cloud.

Several broadband photometric surveys have extended these studies to even larger redshifts (e.g., Hartley et al. 2010; McCracken et al. 2015). Hartley et al., using data from the UKIDSS Ultra Deep Survey, find segregation between passive and star-forming galaxies at $z \lesssim 1.5$ but find consistent clustering properties for both galaxy types at $z \sim 2$.

In the present paper we use the high-quality data of the Advanced Large Homogeneous Area Medium Band Redshift Astronomical survey (ALHAMBRA; Moles et al. 2008; Molino et al. 2014) ${ }^{21}$ to study the clustering segregation of quiescent and star-forming galaxies. ALHAMBRA is very well suited for the analysis of galaxy clustering and segregation studies at very small scales. With a reliable calculation of the projected correlation function we find a clear steepening of the correlation at scales between 0.03 and $0.2 h^{-1} \mathrm{Mpc}$ (Phleps et al. 2006; Coil et al. 2008), specially for the star-forming galaxies. Moreover, its continuous selection function over a large redshift range makes ALHAMBRA an ideal survey for evolution studies. In Arnalte-Mur et al. (2014) (hereafter AM14) the authors presented the results of the evolution of galaxy clustering on scales $r_{p}<10 h^{-1} \mathrm{Mpc}$ for samples selected in luminosity and redshift over $\sim 5 \mathrm{Gyr}$ by

\footnotetext{
${ }^{21}$ http://alhambrasurvey.com
}

means of the projected correlation function $w_{p}\left(r_{p}\right)$. In this paper we use the same statistic to study the evolution of galaxy segregation by spectral type at $0.35<z<1.1$.

Details on the samples used in this analysis are described in Section 2. In Section 3 we introduce the statistic used in our analysis, the projected correlation function, and the methods to obtain reliable estimates of this quantity and to model the results. Finally, in Section 4 we present our results and in Section 5 the conclusions. Throughout the paper we use a fiducial flat $\Lambda$ CDM cosmological model with parameters $\Omega_{M}=0.27, \Omega_{\Lambda}=0.73, \Omega_{b}=0.0458$, and $\sigma_{8}=0.816$ based on the seven-year Wilkinson Microwave Anisotropy Probe (WMAP) results (Komatsu et al. 2011). All the distances used are comoving and are expressed in terms of the Hubble parameter $h \equiv H_{0} / 100 \mathrm{~km} \mathrm{~s}^{-1} \mathrm{Mpc}^{-1}$. Absolute magnitudes are given as $M-5 \log _{10}(h)$.

\section{ALHAMBRA GALAXY SAMPLES}

ALHAMBRA (Moles et al. 2008; Molino et al. 2014) is a project that has imaged seven different areas in the sky through a purposely built set of 20 contiguous, non-overlapping, $310 \AA$ wide filters covering the whole visible range from 3500 to $9700 \AA$ plus the standard near-infrared $J H K_{s}$ filters. The nominal depth $\left(5 \sigma, 3^{\prime \prime}\right.$ aperture $)$ is $I_{A B} \sim 24.5$ and the total sky coverage after masking is $2.381 \mathrm{deg}^{2}$. The final catalog, which is described in Molino et al. (2014), includes over 400,000 galaxies with a photometric redshift accuracy better than $\sigma_{z} /(1+z)=0.014$. Full details on how the accuracy depends on the sample magnitude, galaxy type, and bayesian odds selection limits are given in that work. For the characteristics of the sample that we use in this paper the authors quote a dispersion $\sigma_{\mathrm{NMAD}}<0.014$ and a catastrophic rate $\eta_{1}=0.04 \% .^{22}$ There is no evidence of significantly different behavior for galaxies with spectral energy distributions (SEDs) corresponding to quiescent or star-forming types. Contamination by AGNs is minor (approximately $0.1 \%$ of the sources could correspond to this class, which has not been purged from the ALHAMBRA catalogs) and should be dominated by low-luminosity AGN, which are in many cases fit by strong emission-line galaxies with an approximately correct redshift.

Object detection is performed over a synthetic image created via a combination of ALHAMBRA filters that mimics the Hubble Space Telescope F814W filter (hereafter denoted by I) so that the reference magnitude is directly comparable to other surveys. Photometric redshifts were obtained using the template-fitting code BPZ (Benítez 2000) with an updated set of 11 SED templates as described in Molino et al. (2014). Although a full posterior probability distribution function in redshift $z$ and spectral type $T$ is produced for each object, in this work we take a simpler approach and assign to each galaxy the redshift $z_{b}$ and type $T_{b}$ corresponding to the best fit to its observed photometry. We have checked that the errors induced by the redshift uncertainties, which are partly absorbed by the deprojection technique, are under control as long as we use relatively bright galaxies with good quality photometric redshift determinations. This makes ALHAMBRA a very well-suited catalog: together with the high-resolution photometric redshifts, the abundant imaging allows us both the

\footnotetext{
22 Where $\sigma_{\text {NMAD }}$ is the normalized median absolute deviation and $\eta_{2}$ is defined as the proportion of objects with absolute deviation $|\delta z| /(1+z)>0.2$.
} 


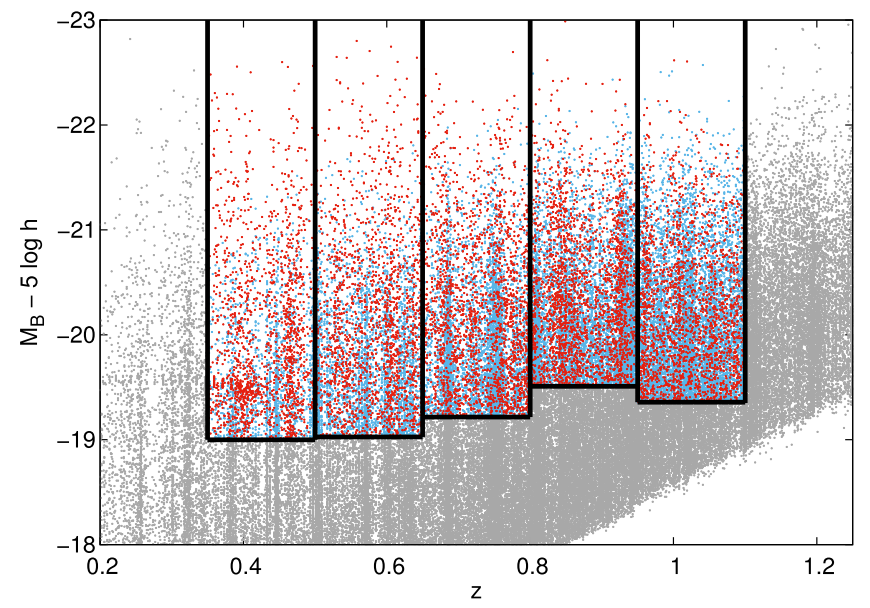

Figure 1. Selected samples with fixed number density in the photometric redshift vs. absolute $B$-band magnitude diagram. The quiescent and starforming galaxy samples are plotted in red and blue, respectively. The solid lines mark the boundaries of our selected samples described in Table 1.

reliable color segregation used in this work as well as a high completeness in the galaxy population at small scale separations, which will be the specific object of a future work.

We have drawn different samples from the ALHAMBRA survey to perform our analysis in a similar way as was done in AM14. First, we cut the magnitude range at $I<24$ where the catalog is photometrically complete (Molino et al. 2014) and we do not expect any significant field-to-field variation in depth. Second, stars are eliminated using the star-galaxy separation method described in Molino et al. (2014). As explained in AM14 the expected contamination by stars in the resulting samples is less than $1 \%$. Finally, we cleanse the catalog using the angular masks defined in AM14, which eliminate regions with less reliable photometry around bright stars or image defects, or very close to the image borders. The sample selected in this way contains 174633 galaxies over an area of $2.381 \mathrm{deg}^{2}$, i.e., with an approximate source density of $7.3 \times 10^{4}$ galaxies per square degree.

Given the ALHAMBRA depth, we divide our sample into five non-overlapping redshift bins. These redshift bins are $\left[0.35,0.5\left[,\left[0.5,0.65\left[,\left[0.65,0.8\left[,\left[0.8,0.95\left[\right.\right.\right.\right.\right.\right.\right.\right.$, and $[0.95,1.1] .^{23}$ In this work we focus on the galaxy spatial segregation by spectral type and use a luminosity selection to obtain a fixed number density. In this way we guarantee that we are comparing similar populations at different redshifts. To select a sample that is complete up to $z=1.1$ we define a threshold magnitude of $M_{B}^{\text {th }}(0)-5 \log (h)=-19.36$ for the highest redshift bin. This limit determines the galaxy number density $\left(\bar{n}=9.35 \times 10^{-3} h^{3} \mathrm{Mpc}^{-3}\right)$ that we will keep constant for the remaining redshift bins; this way our results do not rely on measurements of the luminosity function. This will allow us to study the evolution with redshift of the galaxy spectral segregation. Figure 1 shows the luminosity and redshift selections used in this work. We should remark on the nonmonotonic evolution of the faint limit of our samples with redshift. This effect is not unexpected, as a combination of cosmic variance in the large-scale structure and the artificial redshift peaks that are induced by the photometric redshift methods produce density changes that are observable at the

\footnotetext{
23 Note that the redshift bins used here are different from those in AM14 where overlapping bins were allowed.
}

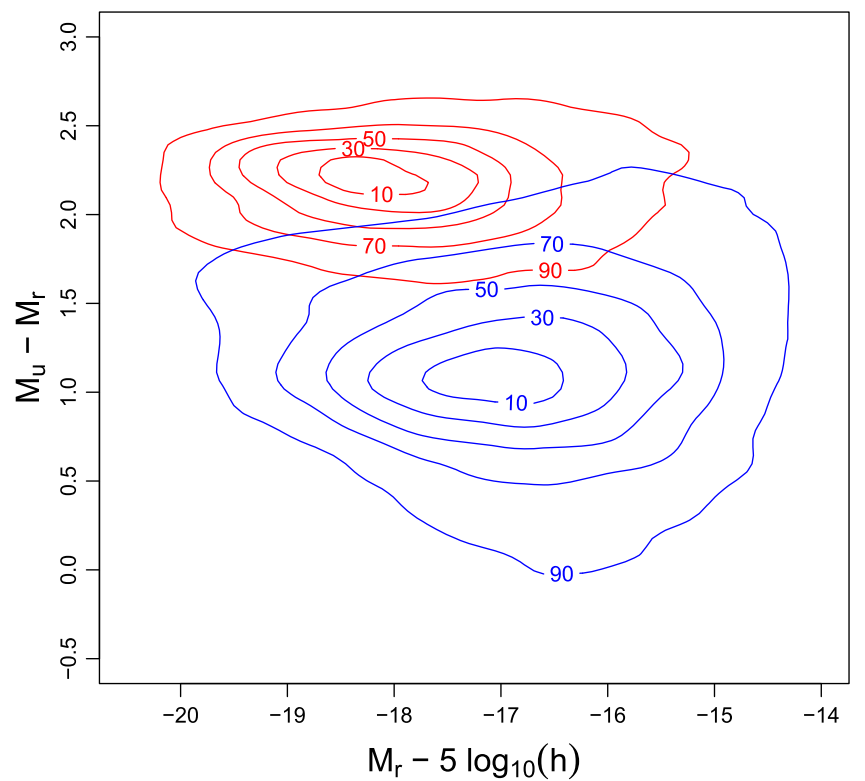

Figure 2. Absolute rest-frame broadband color-magnitude diagram for galaxies with redshifts between 0.35 and 0.75 . Quiescent and star-forming galaxies (selected by their best-fit spectral type) are shown, respectively, as red and blue percentile contours. We have used SDSS absolute magnitudes derived from the ALHAMBRA photometry as described in the text. Our classification by (photometric) spectral type closely matches the usual broadband color selection.

scales we are using. In any case the effect is very small and represents a variation of only $0.1 \mathrm{mag}$ per bin over a monotonic evolution.

We classify our galaxies as "quiescent" and "star-forming" according to the best-fitting template, $T b$, obtained from the BPZ analysis. Templates 1 to 5 correspond to quiescent galaxies, 6 and 7 correspond to star-forming galaxies, and 8 to 11 correspond to starburst galaxies. We consider quiescent galaxies to be those with a template value smaller than 5.5 and star-forming galaxies to be those with a value greater than 5.5. Therefore, we also include in the star-forming category those galaxies classified as starbursts. Note that in the fitting process interpolation between templates is performed.

In a previous work Pović et al. (2013) built a morphological catalog of 22,051 galaxies in ALHAMBRA. We cannot, however, use this catalog as the basis for our analysis as it includes only a small subset of the galaxies in our sample; in its cleanest version it is limited to $A B(\mathrm{~F} 613 \mathrm{~W})<22$ and redshift $z<0.5$ for ellipticals. A cross-check showed that if we identify quiescent galaxies as early-type and star-forming galaxies as late-type, our SED-based classification agrees with the morphological one for over $65 \%$ of the sample. Taking into account that the nominal accuracy of the morphological catalog is $90 \%$, that we are actually using only the objects close to its detection limit and that, as noted in Pović et al. (2013), the relationship between morphological- and color-based classifications is far from being as direct as could naïvely be expected, we consider that these figures prove that the classification is accurate within the expected limits.

In Figure 2 we show how our classification of quiescent and star-forming galaxies performs on a color-luminosity diagram. We plot $M_{r}$ and $M_{u}$, which correspond to the absolute magnitudes in the SDSS rest-frame broadband filters $r$ and $u$ and were estimated from ALHAMBRA data by Stefanon 

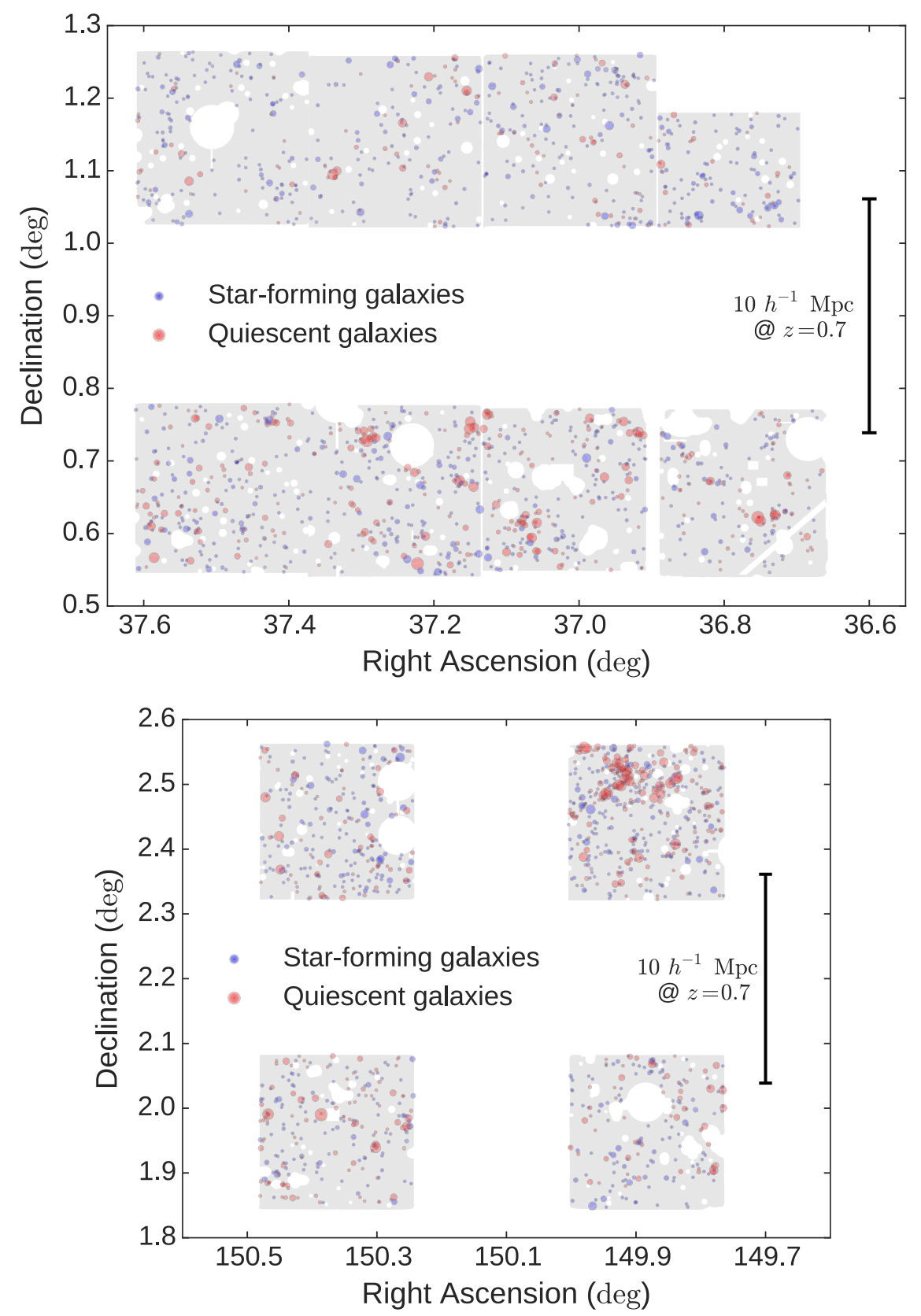

Figure 3. Projection onto the sky of the z0.73 sample $(0.65<z<0.8)$ for two ALHAMBRA fields: ALH-2 (top) and ALH-4/COSMOS (bottom). Galaxies have been colored according to their type: blue circles correspond to star-forming galaxies and red circles to quiescent galaxies with the size of each circle proportional to the luminosity of the corresponding galaxy. North is to the top and east is to the left. The diagram shows the geometry of the ALHAMBRA fields with the angular mask described in the text displayed as a light gray background. The scale of $10 h^{-1} \mathrm{Mpc}$ at $z=0.7$ is indicated as a vertical bar. A heavy concentration of red circles (quiescent galaxies) corresponding to the big coherent structure described in the text is patent in the NW quadrant of the ALH-4/COSMOS field.

(2011) for galaxies with redshift $0.35<z<0.75$ and good quality photometric redshifts. We see how well the ALHAMBRA spectral type classification reproduces the expected behavior (Bell et al. 2004): quiescent galaxies correspond to the "red sequence" in the diagram while star-forming galaxies form the "blue cloud." In addition to the clear segregation in color, we see that quiescent galaxies show on average slightly brighter luminosities than star-forming ones. This shows that our selection by (photometric) spectral type is almost equivalent to a selection in broadband color.

In Figure 3 we show the projection of two fields, ALH-2 and ALH-4/COSMOS, onto the plane of the sky. The coherent superstructure in the ALH-4/COSMOS field at $0.6<z<0.8$ is well appreciated. The skeleton of the structures that form the cosmic web is perfectly delineated by the red quiescent galaxies, while blue star-forming ones tend to populate the field or lower density regions. A similar trend was also visible in the redshift versus R.A. diagram of Guzzo \& The Vipers Team (2013). We will further study this color-density relation (Cucciati et al. 2006) in the following sections by means of the projected correlation function.

Finally, we remove the north-west ALH-4 frame from the analysis (the top right section on the bottom panel of Figure 3). As seen in AM14, there exists an anomalous clustering in the ALH-4 field, which overlaps with the Cosmic Evolution Survey (COSMOS, Scoville et al. 2007b). It is well known that 
Table 1

Characteristics of the Galaxy Samples Used

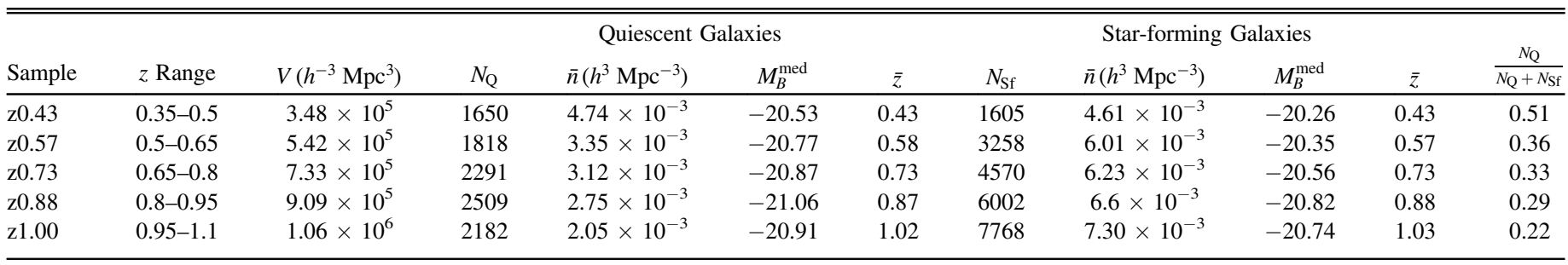

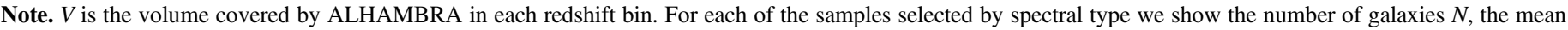

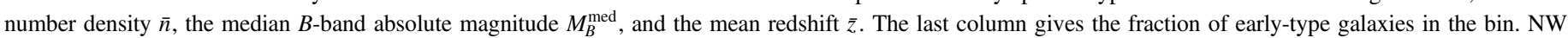
ALH-4 frame is not included.

the COSMOS survey presents higher clustering amplitude than similar surveys (see, e.g., McCracken et al. 2007; de la Torre et al. 2010) due to the presence of large overdense structures in the field (Guzzo et al. 2007; Scoville et al. 2007a). This overdensity of structures is also observed in ALHAMBRA when comparing this peculiar region of the ALH-4 field with the rest of the fields (Molino et al. 2014; Ascaso et al. 2015). In AM14 the authors showed that ALH-4/COSMOS is an outlier in terms of clustering. We have seen that not only does this region introduce anomalies in the measurement of the clustering statistics, but it also affects the error estimation of these statistics. In AM14 the authors also identified ALH-7/ ELAIS-N1 as an outlier field (although the significance of the anomaly was smaller in this case). However, here we do not find any significant change in our results when removing the ALH-7/ELAIS-N1 field, so we keep it in for all our calculations.

For each redshift bin we will analyze the clustering for the full selected population and separately for quiescent and starforming galaxies. Table 1 summarizes the different samples in each redshift bin. Columns $N_{\mathrm{Q}}$ and $N_{\mathrm{Sf}}$ are the number of quiescent and star-forming galaxies, respectively, and the last column is the fraction of quiescent galaxies in each of the redshift subsamples.

We see that the fraction of quiescent galaxies decreases with redshift. This behavior is expected qualitatively, as blue starforming galaxies are dominant at earlier cosmic times while red quiescent galaxies appear late once star formation stops. This trend was also observed in a similar redshift range by e.g., Zucca et al. (2009) for the zCOSMOS 10k bright sample. They found that the population of bright late-type galaxies becomes dominant at higher redshifts and therefore the fraction of earlytype galaxies decreases with redshift accordingly.

\section{METHODS}

The method used for the correlation analysis of our data follows closely the one used in AM14, where it is discussed in detail. We present here a summary of the methods and some points where the details differ. We estimate the correlation function using the estimator proposed by Landy \& Szalay (1993) and the projected correlation function to recover realspace clustering from our photometric redshift catalogs as described by Davis \& Peebles (1983) and Arnalte-Mur et al. (2009). We use the delete-one jackknife method for the error estimation and linear regression to fit the different model correlation functions to our data.

\subsection{Estimation of the Projected Correlation Function}

The method introduced by Davis \& Peebles is based on the decomposition of pair separations in distances parallel and perpendicular to the line of sight, $\left(r_{\|}, r_{p}\right)$. Given two galaxies, if we define their radial vectors to the observer as $s_{1}$ and $s_{2}$, then their separation vector is $s \equiv s_{1}-s_{2}$ and the line of sight vector is $\boldsymbol{l} \equiv \boldsymbol{s}_{1}+\boldsymbol{s}_{2}$. From these we can now calculate the transverse and radial distances as

$$
r_{\|} \equiv \frac{|s \cdot l|}{|l|}, r_{p} \equiv \sqrt{s \cdot s-r_{\|}^{2}} .
$$

Once $\left(r_{\|}, r_{p}\right)$ are defined for each galaxy pair we can proceed to calculate the two-dimensional correlation function, $\xi\left(r_{\|}, r_{p}\right)$ in an analogous way to $\xi(r)$ (Martínez \& Saar 2002). With this method, for every galaxy pair we define a plane passing through the observer and containing the vectors $s_{1}$ and $s_{2}$. Therefore, we only need to assume isotropy in the plane that is perpendicular to the line of sight.

To estimate the projected correlation function we need a random Poisson catalog with the same selection function as our data. We create this auxiliary catalog in each case using the software MANGLE (Hamilton \& Tegmark 2004; Swanson et al. 2008) to apply the angular selection mask defined in AM14. In addition, the random points are distributed so that they follow the redshift distribution of each of the galaxy samples used (see Section 2). We estimate the two-dimensional two-point correlation function as (Landy \& Szalay 1993)

$$
\hat{\xi}\left(r_{\|}, r_{p}\right)=1+\left(\frac{N_{R}}{N_{D}}\right)^{2} \frac{D D\left(r_{\|}, r_{p}\right)}{R R\left(r_{\|}, r_{p}\right)}-2 \frac{N_{R}}{N_{D}} \frac{D R\left(r_{\|}, r_{p}\right)}{R R\left(r_{\|}, r_{p}\right)},
$$

where $D D\left(r_{\|}, r_{p}\right), D R\left(r_{\|}, r_{p}\right)$, and $R R\left(r_{\|}, r_{p}\right)$ correspond to pairs of points with transverse separations in the interval $\left[r_{p}, r_{p}+d r_{p}\right]$ and radial separations in the interval $\left[r_{\|}, r_{\|}+d r_{\|}\right]$. DD counts pairs of points in the data catalog, $R R$ counts pairs in the random Poisson catalog, and $D R$ counts crossed pairs between a point in the data catalog and a point in the Poisson catalog. $N_{D}$ is the number of points in the data catalog and $N_{R}\left(=20 N_{D}\right)$ is the number of points used in our random Poisson catalog.

We can define the projected correlation function as

$$
w_{p}\left(r_{p}\right)=2 \int_{0}^{\infty} \hat{\xi}\left(r_{\|}, r_{p}\right) d r_{\|}
$$

As $w_{p}$ depends only on $r_{p}$ and the angle between any pair of points is small, it will not be significantly affected by redshift 
errors as these will mainly produce shifts in $r_{\|}$. For computational reasons we have to fix a finite upper limit, $r_{\|, \max }$, for the integral in Equation (3). The authors showed in AM14 that the optimal value for our samples is $r_{\|, \max }=200 h^{-1} \mathrm{Mpc}$.

We further correct our measured $w_{p}\left(r_{p}\right)$ for the bias introduced by the integral constraint (Peebles 1980). This effect arises because we are measuring the correlation function with respect to the mean density of a sample instead of the global mean of the parent population. We base our correction on the effect of the integral constraint on the 3D correlation function $\xi(r)$. This function is biased to first order as

$$
\xi(r)=\xi^{\text {true }}(r)-K,
$$

where $K$ is the integral constraint term. Using Equation (3) the effect on $w_{p}\left(r_{p}\right)$ is then

$$
w_{p}^{\text {true }}\left(r_{p}, r_{\|, \max }\right)=w_{p}\left(r_{p}, r_{\|, \max }\right)+2 K r_{\|, \max } .
$$

Given a model correlation function, $K$ can be estimated as (Roche et al. 1999)

$$
K \simeq \frac{\sum_{i} R R\left(r_{i}\right) \xi^{\text {model }}\left(r_{i}\right)}{\sum_{i} R R\left(r_{i}\right)}=\frac{\sum_{i} R R\left(r_{i}\right) \xi^{\text {model }}\left(r_{i}\right)}{N_{R}\left(N_{R}-1\right)} .
$$

We proceed in an iterative way to introduce this correction for each of our samples. We first fit our original $w_{p}\left(r_{p}\right)$ measurements to a double power-law model, $A \cdot x^{\beta}+C \cdot x^{\delta}$. We use the model $\xi(r)$ obtained from this fit to estimate $K$ using Equation (6) and obtain our corrected values of $w_{p}\left(r_{p}\right)$ from Equation (5). We use the corrected values to perform the model fits described in Sections 4.1 and 4.2, and for all the results reported in Section 4. In any case, the effect of the integral constraint in our measurements is always much smaller than the statistical errors.

To estimate the correlation function errors for each bin in $r_{p}$, we used the jackknife method (see, e.g., Norberg et al. 2009). We divided our volume in $N_{\text {jack }}=47$ equal sub-volumes, corresponding to the individual ALHAMBRA frames (see Molino et al. 2014), and constructed our jackknife samples omitting one sub-volume at a time. We repeated the full calculation of $w_{p}\left(r_{p}\right)$ (including the integral constraint correction) for each of these samples. Denoting by $w_{p i}^{k}$ the correlation function obtained for bin $i$ in the jackknife sample $k$, the covariance matrix of the projected correlation function is then

$$
\Sigma_{i j}=\frac{N_{\text {jack }}-1}{N_{\text {jack }}} \sum_{k=1}^{N_{\text {jack }}}\left(w_{p}^{k}\left(r_{i}\right)-\bar{w}_{p}\left(r_{i}\right)\right) \cdot\left(w_{p}^{k}\left(r_{j}\right)-\bar{w}_{p}\left(r_{j}\right)\right),
$$

where $\bar{w}_{p i}$ is the average of the values obtained for bin $i$. The errors for individual data points (shown as error bar in the plots) are obtained from the diagonal terms of the covariance matrix as

$$
\sigma_{i}=\sqrt{\Sigma_{i i}}
$$

\section{RESULTS AND DISCUSSION}

In this section we first present the results of the calculation of the projected correlation function $w_{p}\left(r_{p}\right)$ for the different samples described in Section 2. This is done in Section 4.1. We
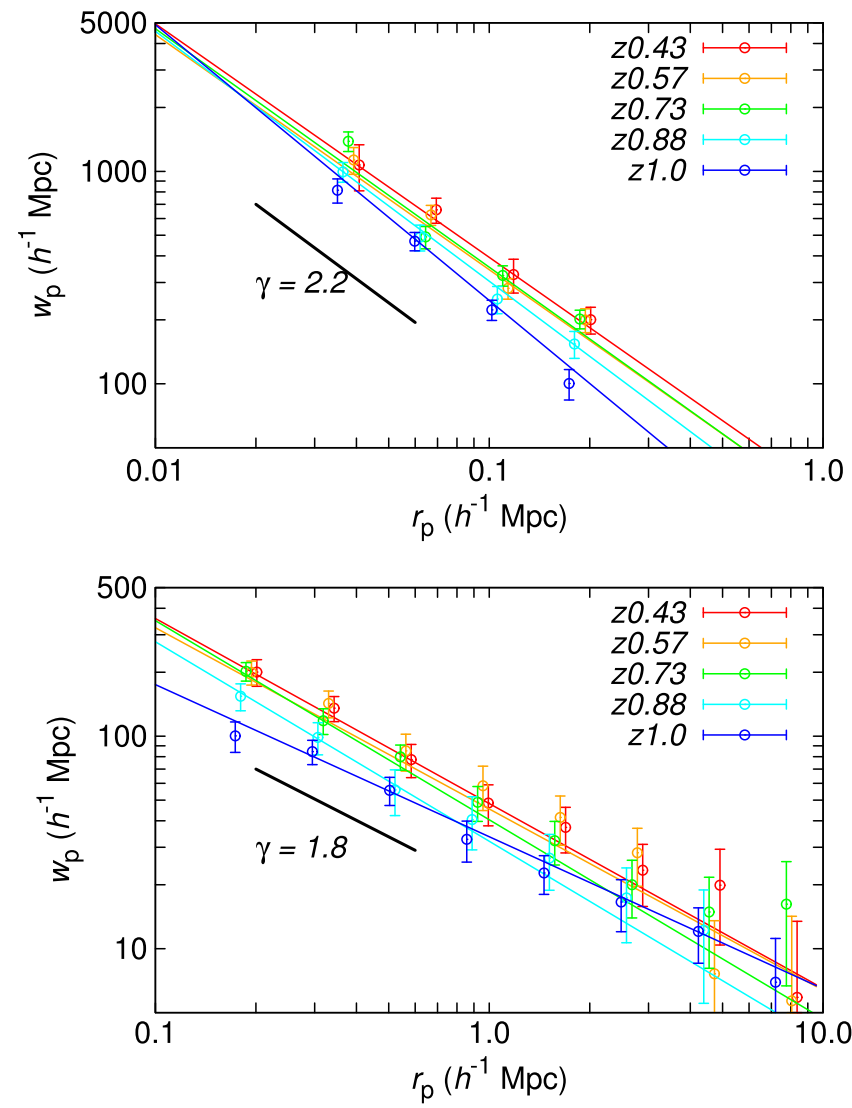

Figure 4. Projected correlation function for the full population sample in each of the redshift bins (points with error bars). Top: small scales $\left(0.03<r_{p}<0.2\right)$. Bottom: large scales $\left(0.2<r_{p}<10.0\right)$. Error bars are calculated with the delete-one jackknife method and values at the same $r_{p}$ are shifted for clarity. Solid lines with matching colors show the best-fit power law in each case. The black segment represents the mean slope of the curves.

also present the analysis of the bias (Section 4.2). The calculation has been performed for scales from 0.03 to 10.0 $h^{-1} \mathrm{Mpc}$ for the projected correlation function and from 1.0 to $10.0 h^{-1} \mathrm{Mpc}$ for the bias. Figure 4 shows the projected correlation function for the full samples. The first remarkable result that deserves to be pointed out is a clear change of the slope of the $w_{p}\left(r_{p}\right)$ functions around $r_{p} \sim 0.2 h^{-1} \mathrm{Mpc}$, as already mentioned by Coil et al. (2006, 2008).

In this section we compare our results with previous works that studied the galaxy clustering and its dependence on spectral type or color in the redshift range $z \in[0,1]$ as mentioned in the introduction. Given the luminosity selection of our sample (see Section 2), in each case we use for comparison the published results for volume-limited samples with number density closest to $n=10^{-2} h^{3} \mathrm{Mpc}^{-3}$. The number density of the samples shown in our comparisons are within $20 \%$ of this figure with two exceptions: the PRIMUS sample at $z \simeq 0.4$ (with number density of $n=1.6 \times 10^{-2} h^{3} \mathrm{Mpc}^{-3}$, Skibba et al. 2014) and the VIPERS sample at $z \simeq 0.6$ (with number density of $n=0.33 \times 10^{-2} h^{3} \mathrm{Mpc}^{-3}$, Marulli et al. 2013). In the case of Meneux et al. (2006), they use a flux-limited sample resulting in a evolving number density with redshift in the range $n=0.33-1.2 \times 10^{-2} h^{3} \mathrm{Mpc}^{-3}$. 


\subsection{Power-law Modeling}

Power laws are simple and widely used models that describe the correlation function of the galaxy distributions, as they provide a very good approximation over a large range of scales with only two free parameters. The observed change of the slope mentioned above forced us to model the projected correlation function $w_{p}$ by means of two power laws, one that fits the function at small scales and the other one at large scales. A similar treatment was done by Coil et al. (2006) in their analysis of the clustering in the DEEP2 survey at $z=1$. The departure from power-law behavior at small scales can be explained naturally in the framework of the halo occupation distribution (HOD) model that considers the contribution to the correlation function of pairs within the same halo (one-halo term), which is dominant at short scales, and the transition to the regime where the function is dominated by pairs from different halos (two-halo term) at large scales. We will present HOD fits to the ALHAMBRA data in a separate paper. Therefore, we fit two power laws as

$$
w_{p}^{s}\left(r_{p}\right)=A r_{p}^{\beta}, \quad \text { if } r_{p} \leqslant r_{s}
$$

for the small scales, and

$$
w_{p}^{l}\left(r_{p}\right)=C r_{p}^{\delta}, \quad \text { if } r_{p} \geqslant r_{s}
$$

for the large ones. We fix value $r_{s} \simeq 0.2 h^{-1}$ Mpc. An abrupt change in the projected correlation function has also been detected at this scale by Phleps et al. (2006) for the blue galaxies of the COMBO-17 sample. $A, \beta, C$, and $\delta$ are the free parameters. We treat each power law independently and express them in terms of the equivalent model for the 3D correlation function $\xi$.

$$
\xi^{\mathrm{pl}}(r)=\left(\frac{r}{r_{0}}\right)^{-\gamma} .
$$

$A$ and $\beta$ (analogously, $C$ and $\delta$ ) can be related to the parameters $\gamma$ (power-law index) and $r_{0}$ (correlation length) as shown in Davis \& Peebles (1983):

$$
A=r_{0}^{\gamma} \frac{\Gamma(0.5) \Gamma[0.5(\gamma-1)]}{\Gamma(0.5 \gamma)}, \beta=1-\gamma
$$

We have performed the fitting of this model to our data using a standard $\chi^{2}$ method, by minimizing the quantity

$$
\begin{aligned}
\chi^{2}\left(r_{0}, \gamma\right)= & \sum_{i=1}^{N_{\text {bins }}} \sum_{j=1}^{N_{\text {bins }}}\left(w_{p}\left(r_{i}\right)-w_{p}^{p w}\left(r_{i}\right)\right) \\
& \cdot \sum_{i j}^{-1} \cdot\left(w_{p}\left(r_{j}\right)-w_{p}^{p w}\left(r_{j}\right)\right),
\end{aligned}
$$

where $\Sigma$ is the covariance matrix. We fit this model to our data at scales $0.03 \leqslant r_{p} \leqslant 0.2 h^{-1} \mathrm{Mpc}$ and $0.2 \leqslant r_{p} \leqslant 10.0 h^{-1} \mathrm{Mpc}$ for each sample using the covariance matrix computed from Equation (7) to obtain the best-fit values of $r_{0}, \gamma$ and their uncertainties (see Table 2). This fitting has been performed using the POWERFIT code developed by Matthews \& Newman (2012).

We must remark that the statistical errors of the correlation function at different separations $r_{p}$ are heavily correlated because a given large-scale structure adds pairs at many different distances. The higher the values of the off-diagonal terms of the covariance matrix, the stronger the correlations between the errors. When this happens the best-fit parameters $r_{0}$ and $\gamma$ might be affected as has been illustrated by Zehavi et al. (2004) for the SDSS survey. One could ignore the error correlations and use only the diagonal terms, but this is not justified if these terms are dominant. The parameters of the fits are listed in Table 2.

\subsubsection{Full Samples}

Figure 4 (top panel) shows the measurements of the projected correlation function $w_{p}\left(r_{p}\right)$ for the full samples at the small scales $\left(0.03 \leqslant r_{p} \leqslant 0.2 h^{-1} \mathrm{Mpc}\right)$. The bottom panel shows the same function for large scales $\left(0.2 \leqslant r_{p} \leqslant 10.0 h^{-1}\right.$ $\mathrm{Mpc}$ ). Looking at both diagrams, we confirm the rise of the correlation function at small scales already detected by Coil et al. (2008) with values of $\gamma \sim 2.2$ (for the slope of the threedimensional (3D) correlation function). We can also appreciate in the top panel of Figure 4 that the correlation functions are steeper for the high-redshift samples with values of $\gamma$ increasing from $\sim 2.1$ for the closest redshift bin $(z \sim 0.4)$ to $\sim 2.3$ for the farthest $(z \sim 1)$. The correlation length significantly decreases with increasing redshift (see also Table 2).

For the large scales $0.2 \leqslant r_{p} \leqslant 10.0 h^{-1} \mathrm{Mpc}$, the slope of the correlation function is rather constant for all samples with values around $\gamma=1.8$, while again the correlation length decreases with redshift from $r_{0}=4.1 \pm 0.5$ for $z \sim 0.4$ to $r_{0}=3.5 \pm 0.3$ for $z \sim 1$. The evolution of the amplitude indicates that the change in clustering is mainly driven by the overall growth of structure in the matter density field. As we use for the fits the scales $0.2<r_{p}<10.0 h^{-1} \mathrm{Mpc}$ (the 2-halo term becoming important at scales $r_{p}>1.0 h^{-1} \mathrm{Mpc}$ ) the fact that the slope $\gamma$ does not significantly change also implies that the 2-halo contribution for this population does not significantly change its profile over this redshift interval. All these effects were studied in detail in AM14 and extended to samples with different luminosities (see e.g., their Figure 7). We have seen that this is only broken at shorter scales where the curve presents slightly higher values.

The overall trend can be visualized in Figure 5 where we show the evolution of the best-fit parameters of the 3D correlation function $\xi(r)$ for small and large scales in the full population samples. Despite the great uncertainties, the diagram shows evolution with $r_{0}$ decreasing for both scale ranges as redshift grows. In addition, at small scales, the slope $\gamma$ also increases with redshift. The evolution at large scales of the correlation length extrapolates well to lower redshift with the value reported by Zehavi et al. (2011) for the SDSS and by Madgwick et al. (2003) for the $2 \mathrm{dF}$ galaxy redshift survey. Zehavi et al. (2011) analyzed the SDSS Main catalog by means of the projected correlation function. They obtained values for the parameters $r_{0}$ and $\gamma$ by the same method used here, over the scale range $0.1<r_{p}<50 \mathrm{~h}^{-1} \mathrm{Mpc}$. The values correspond to the galaxies selected in the luminosity bin $-20<M_{r}<-19$ and $0.027<z<0.064$, with a number density $\left(n=10.04 \times 10^{-3} h^{3} \mathrm{Mpc}^{-3}\right)$ and typical luminosity $\left(L^{\text {med }} / L^{\star}=0.4\right)$ similar to the ALHAMBRA sample used in this work, so qualitatively this is a valid comparison. As we can see in Figure 5 the slope of the correlation function for the full SSDS main sample is $\gamma=1.78 \pm 0.02$ compatible within one $\sigma$ with the values obtained for the ALHAMBRA survey at higher redshift within the range of large scales analyzed here and the correlation length $r_{0}=4.89 \pm 0.26$ follows the evolutionary trend delineated by the ALHAMBRA higher 
Table 2

Results of the Different Fits to $w\left(r_{p}\right)$ : Power-law and Bias Models

\begin{tabular}{|c|c|c|c|c|c|}
\hline \multirow[t]{2}{*}{ Sample } & \multicolumn{5}{|c|}{ Full Population } \\
\hline & $r_{0}^{s}$ & $\gamma^{s}$ & $r_{0}^{l}$ & $\gamma^{l}$ & $b$ \\
\hline $\mathrm{z} 0.43$ & $3.1 \pm 0.6$ & $2.1 \pm 0.13$ & $4.1 \pm 0.5$ & $1.87 \pm 0.12$ & $1.21 \pm 0.14$ \\
\hline $\mathrm{z} 0.57$ & $2.9 \pm 0.4$ & $2.11 \pm 0.1$ & $4 \pm 0.5$ & $1.85 \pm 0.1$ & $1.23 \pm 0.17$ \\
\hline $\mathrm{z} 0.88$ & $2.5 \pm 0.4$ & $2.18 \pm 0.1$ & $3.2 \pm 0.7$ & $1.94 \pm 0.15$ & $1.2 \pm 0.5$ \\
\hline $\mathrm{z} 1.00$ & $2 \pm 0.3$ & $2.3 \pm 0.11$ & $3.5 \pm 0.3$ & $1.72 \pm 0.06$ & $1.3 \pm 0.13$ \\
\hline $\mathrm{z} 0.57$ & $2.3 \pm 0.5$ & $2.62 \pm 0.18$ & $5.4 \pm 0.8$ & $1.85 \pm 0.11$ & $1.8 \pm 0.2$ \\
\hline $\mathrm{z} 0.73$ & $3.6 \pm 0.7$ & $2.29 \pm 0.14$ & $4.3 \pm 0.7$ & $2.15 \pm 0.16$ & $1.4 \pm 0.2$ \\
\hline $\mathrm{z} 0.88$ & $4 \pm 0.9$ & $2.25 \pm 0.13$ & $4.2 \pm 0.8$ & $2.14 \pm 0.17$ & $1.6 \pm 0.3$ \\
\hline \multirow[t]{2}{*}{$\mathrm{z} 1.00$} & $3.5 \pm 0.9$ & $2.28 \pm 0.16$ & $4.8 \pm 0.8$ & $1.8 \pm 0.13$ & $1.9 \pm 0.3$ \\
\hline & \multicolumn{5}{|c|}{ Star-forming Galaxies } \\
\hline $\mathrm{z} 0.88$ & $1.8 \pm 0.5$ & $2.3 \pm 0.2$ & $3 \pm 0.4$ & $1.7 \pm 0.12$ & $1.2 \pm 0.4$ \\
\hline $\mathrm{z} 1.00$ & $1.7 \pm 0.3$ & $2.34 \pm 0.13$ & $3.2 \pm 0.3$ & $1.69 \pm 0.09$ & $1.25 \pm 0.13$ \\
\hline
\end{tabular}

Note. Results of the fits of the power-law model and the bias model to the data for each of our samples. $r_{0}^{s}$ and $\gamma^{s}$ correspond to the scales $0.03<r_{p}<0.2 h^{-1} \mathrm{Mpc}$, and $r_{0}^{l}$ and $\gamma^{l}$ to the scales $0.2<r_{p}<10.0 h^{-1} \mathrm{Mpc}$. These parameters have been calculated using the methods described in Sections 4.1 and 4.2.

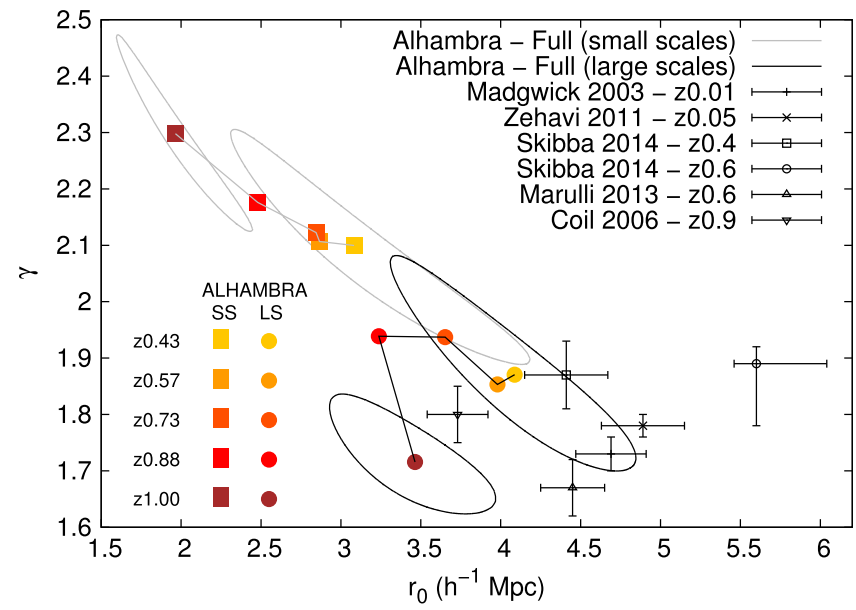

Figure 5. Parameters $r_{0}, \gamma$ obtained from the power-law fit to the projected correlation functions of our full population samples. In black, the $1 \sigma$ confidence regions of the large scales fit $\left(0.2<r_{p}<10.0 h^{-1} \mathrm{Mpc}\right)$ and in gray, the $1 \sigma$ confidence regions of the small scales fit $\left(0.03<r_{p}<0.2 h^{-1} \mathrm{Mpc}\right)$. For clarity we show only the regions for the first and last redshift bin. Lines link the best-fit results for each sample accross different redshift bins. For comparison we show as points with error bars the results of Madgwick et al. (2003) (2dF), Zehavi et al. (2011) (SDSS), Coil et al. (2006) (DEEP2), Marulli et al. (2013) (VIPERS), and Skibba et al. (2014) (PRIMUS) (see the text for details). The parameters and their 1-sigma variation have been calculated using the method described in Section 4.

redshift samples: $r_{0}$ increases at lower redshifts. Very similar results have been obtained by Madgwick et al. (2003) for the 2dFGRS with $\gamma=1.73 \pm 0.03$ and $r_{0}=4.69 \pm 0.22$ within the range $0.2<r_{p}<20.0 h^{-1} \mathrm{Mpc}$ in the redshift interval $0.01<z<0.015$. At larger redshift our results can be compared with the ones reported by Skibba et al. (2014) for the PRIMUS survey. They have analyzed two bins of redshift $0.2<z<0.5$ and $0.5<z<1$ with $M_{g}<-19$. In Figure 5 we have displayed their results for the correlation function parameters. We also plot a point corresponding to the VIMOS Public Extragalactic Redshift Survey (VIPERS) from Marulli et al. (2013) and another point corresponding to the DEEP2 survey from Coil et al. (2006). All these results for the three high-redshift surveys show perfect agreement with our own ALHAMBRA results.

It is important to understand the correlation between parameters $\gamma$ and $r_{0}$, as its interpretation can be delicate. If, for instance, $\gamma$ grows with the redshift of the sample, $r_{0}$ will tend to reduce its value, as $\xi(r)=1$ for shorter distances, as it can be appreciated in the top-left points (short $r_{p}$ scales) displayed in Figure 5 We must have this in mind for a proper understanding of our results. On the other hand, the decrease of $r_{0}$ with increasing redshift when $\gamma$ does not change, as we find in bottom-right points (large scales) in Figure 5, can be interpreted as a self-similar growth of the structure at the calculated scales. This effect is specially reflected in the tilt of the confidence ellipses in Figure 5, which shows the negative correlation between $r_{0}$ and $\gamma$.

\subsubsection{Segregated Samples}

Figure 6 shows the projected correlation function $w_{p}\left(r_{p}\right)$ for the quiescent and star-forming galaxies at the five redshift bins compared with the full population. As expected, the full population result occupies an intermediate position at low redshift but evolves with redshift toward star-forming positions. This is expected due to the higher abundance of the latter in our samples, specially at high redshift. A visual inspection of Figure 6 suggests that the projected correlation function shows the double slope corresponding to the 1-halo and the 2-halo terms specially for the star-forming galaxies, due to their tendency to cluster in lower mass halos with smaller virial radii (Seljak 2000).

Quiescent galaxies show a higher clustering at every redshift bin. To study the change of the clustering properties with 


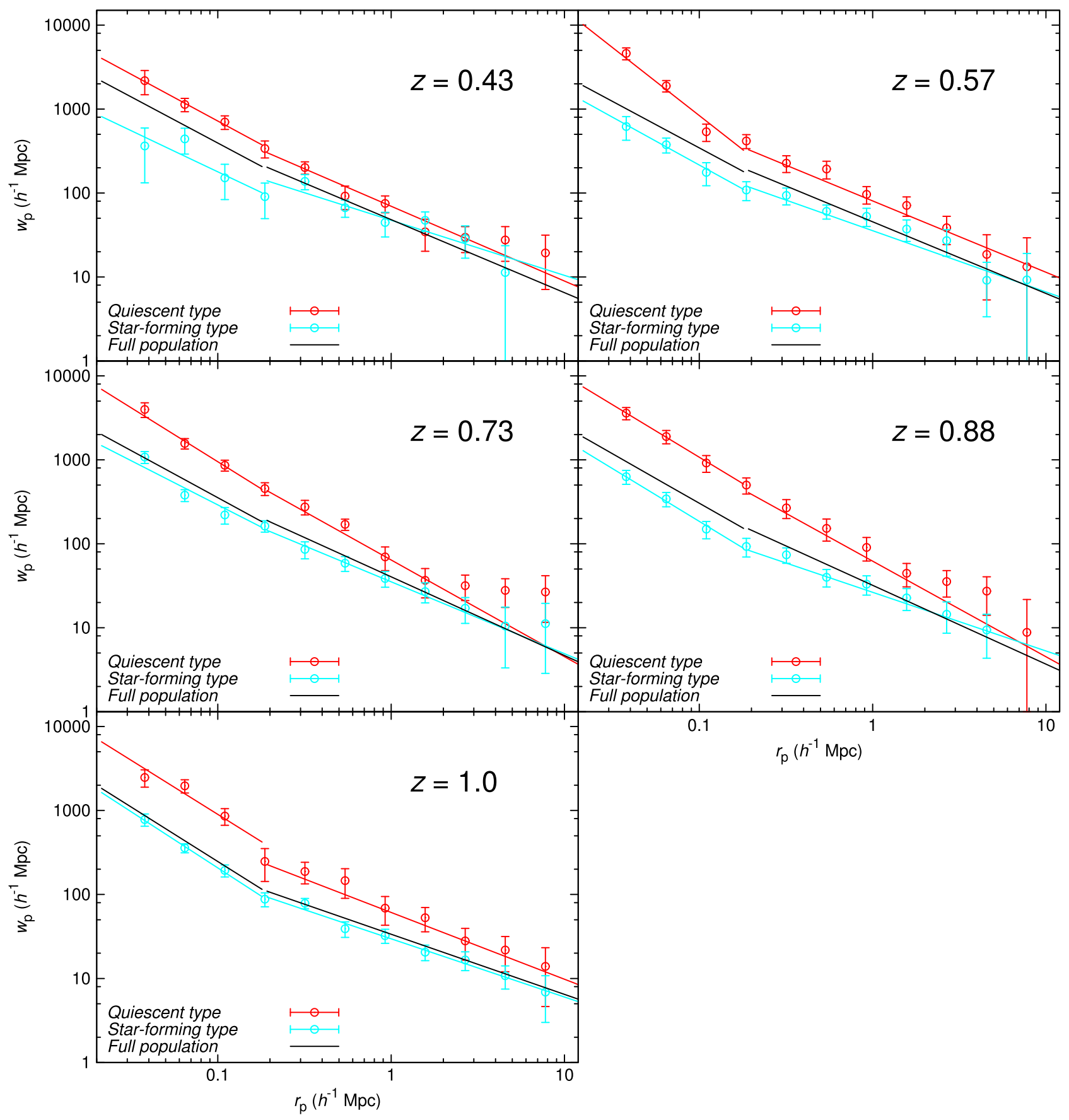

Figure 6. Projected correlation functions for quiescent (red) and star-forming (blue) galaxies (points with error bars). Solid lines with matching colors show the best-fit power law in each case. For reference we also show the results for the full population with the continuous black line. From top to bottom, left to right, the five redshift bins: (0.35<z<0.5), $(0.5<z<0.65),(0.65<z<0.8),(0.8<z<0.95)$, and $(0.95<z<1.1)$. Error bars are calculated with the delete-one jackknife method.

redshift and spectral type, we fit the projected correlation function $w_{p}\left(r_{p}\right)$ of each sample with a power-law model using the method described above.

The amplitude of their correlation functions as well as their slope is higher than that for the star-forming galaxies in all cases. As for the full population we have modeled the correlation function with two different power laws at scales larger and smaller than $r_{p}=0.2 h^{-1} \mathrm{Mpc}$. Star-forming galaxies show for all redshift bins a clear rise in their correlation function at small separations. As mentioned in the introduction, Coil et al. (2008) found the same result for the bright blue galaxies of the DEEP2 galaxy redshift survey. They found that the effect is more pronounced at higher redshift corresponding to brighter galaxies. For the quiescent galaxies we would have fitted a single power law for the whole range, in particular for some redshift bins. However, we have proceeded in the same way for the two galaxy types to simplify the analysis of the segregation. The comparison of the best-fit model with the data in each case is shown in Figure 6 and we see an excellent agreement in all cases. The parameters obtained from the fits are listed in Table 2.

As we have done for the full population, to visualize if there is any evolution of the correlation function parameters we show the diagram of $\gamma$ versus $r_{0}$ in Figures 7 and 8 for the segregated 


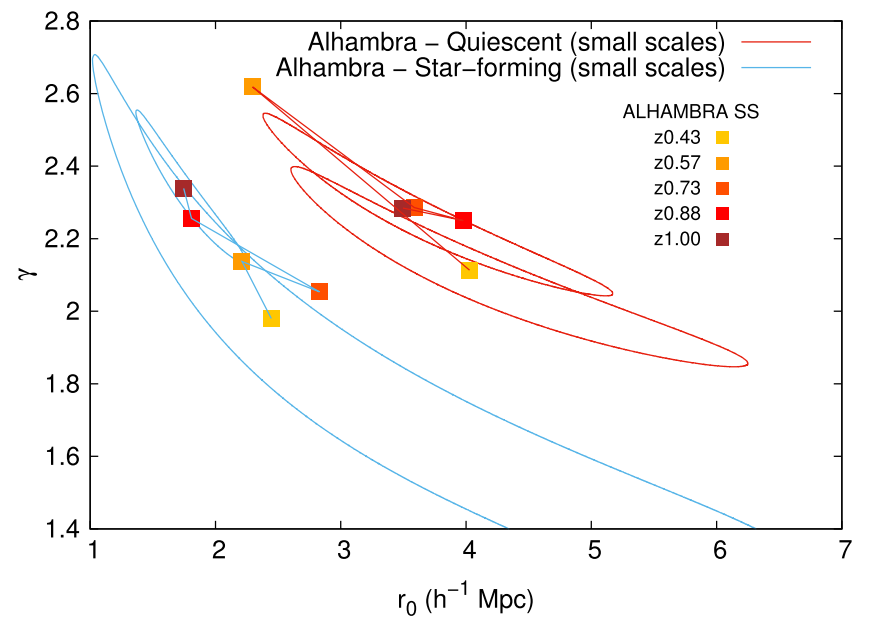

Figure 7. Parameters $r_{0}, \gamma$ obtained from the power-law fit to the projected correlation functions of our spectral segregated samples for the small scales $\left(r_{p} \leqslant 0.2 h^{-1}\right)$. In red, the $1 \sigma$ confidence regions of the quiescent galaxies fit and in blue, the $1 \sigma$ confidence regions of the star-forming galaxies fit. For clarity we show only the regions for the first and last redshift bin. Lines link the best-fit results for each sample across different redshift bins. The parameters and their 1-sigma variation have been calculated using the method described in Section 4.1.

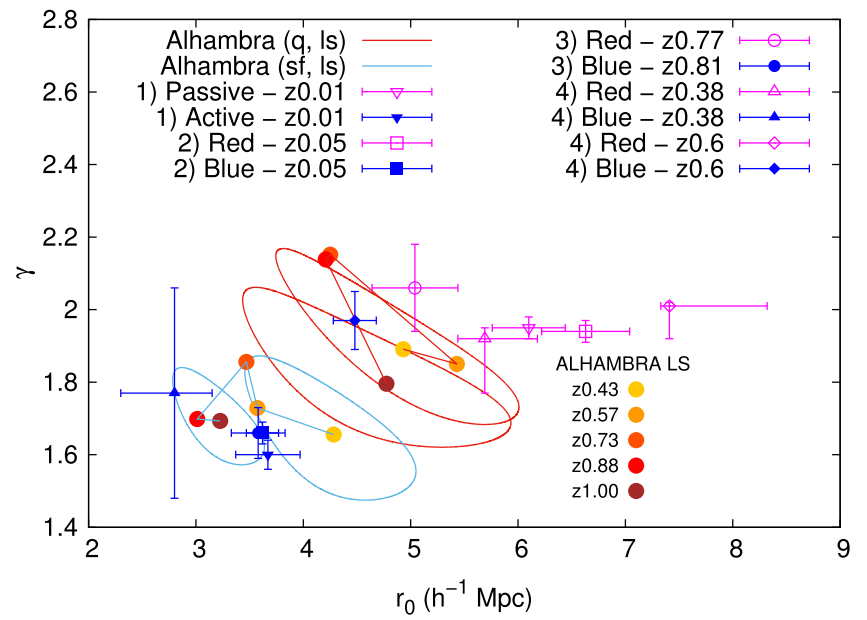

Figure 8. Parameters $r_{0}, \gamma$ obtained from the power-law fit to the projected correlation functions of our spectral segregated samples. In red, the $1 \sigma$ confidence regions of the quiescent galaxies fit and in blue, the $1 \sigma$ confidence regions of the star-forming galaxies fit. For clarity we show only the regions for the first and last redshift bin. Lines link the best-fit results for each sample across different redshift bins. For comparison we show as points with error bars the results of Zehavi et al. (2011) and Coil et al. (2008) (see the text for details). The parameters and their 1-sigma variation have been calculated using the method described in Section 4.1. For comparison we plot the results obtained by (1) Madgwick et al. (2003) (2dF) at $z \sim 0.01$, (2) Zehavi et al. (2011) (SDSS) at $z \sim 0.05$, (3) Coil et al. (2008) (DEEP2) at $z \sim 0.9$ and (4) Skibba et al. (2014) (PRIMUS) at $z \sim 0.38$ and $z \sim 0.6$.

populations with the corresponding confidence regions, separated in the two scale regimes. In both cases (short and large scales) the parameter space occupied by quiescent galaxies can be clearly distinguished from the space occupied by star-forming galaxies, the first ones showing a larger correlation length for both scaling ranges with the difference between both types well over $3 \sigma$ for small scales and about $2 \sigma$ for large scales.

At short scales the exponent of the correlation function $\gamma$ is similar for both galaxy types with values around $\gamma \sim 2.2$
(Figure 7). The correlation length for star-forming galaxies varies roughly in the range $r_{0}=[2,3] h^{-1} \mathrm{Mpc}$. A visual hint of evolution could be appreciated for the star-forming galaxies, with steeper correlation functions (and lower correlation lengths) for higher redshifts; nevertheless, given the large error bars (see Table 2), this trend is not really significant. The parameters of the correlation function for quiescent galaxies at small scales do not show any evolution at all with values for $\gamma$ in the range $[2.1,2.3]$ and correlation lengths in the range $r_{0}=[3.5,4.0] h^{-1} \mathrm{Mpc}$ for all redshift bins except for the second bin $(z=0.57)$, which displays a higher value of the exponent $\gamma$ and smaller $r_{0}$. The ALHAMBRA survey has allowed us to measure the behavior of the clustering properties of the segregated samples at these very short scales. These scales had not been previously studied with the detail that we are showing here because other samples cannot reliably estimate the correlation function at $r_{p}<0.1 h^{-1} \mathrm{Mpc}$ because they are not deep enough or dense enough at these distance due, for example, to fiber collisions in the case of spectroscopic surveys (Guo et al. 2012).

Figure 8 shows the same results at scales $0.2<r_{p}<10.0 h^{-1} \mathrm{Mpc}$. For this scale range we can compare with the results from other authors. We see again that the regions of parameter space occupied in the diagram for quiescent and star-forming galaxies are different. Star-forming galaxies present both lower exponent $\gamma$ and lower correlation length $r_{0}$ than quiescent galaxies. These differences are significant at the $2 \sigma$ level. The value of $\gamma$, exponent of the correlation function, is roughly constant for all redshift bins and is $\sim 1.7$. A hint of evolution can be seen in the correlation length since $r_{0}$ decreases from $r_{0} \sim 4.3$ to $r_{0} \sim 3 h^{-1} \mathrm{Mpc}$ with increasing redshift, which corresponds to a $\sim 2 \sigma$ change in $r_{0}$. The values of the correlation function parameters reported by other authors for different samples at lower and similar redshift are compatible with the ALHAMBRA results shown here. In the diagram we see that our fits are consistent with the points corresponding to the correlation function parameters of the active galaxies from the 2dFGRS (Madgwick et al. 2003) at $z \sim 0.01$, a blue subsample drawn from the SDSS-main (Zehavi et al. 2011) at $z \sim 0.05$, a blue population of the DEEP2 redshift survey (Coil et al. 2008) at $z \sim 0.9$, and the blue sample from the PRIMUS survey (Skibba et al. 2014) at $z \sim 0.4$. This result also agrees with the qualitative behavior of the evolution of the correlation length reported by Meneux et al. (2006) from the VVDS sample in which they conclude that the clustering amplitude of the late-type star-forming galaxies remains roughly constant since $z \sim 1.5$, although they found a slight rise of this amplitude at their larger redshift bin $1.2<z<2.0$. However, one should bear in mind that Meneux et al. (2006) use a flux-limited sample, so this evolution may be affected by the change in luminosity of the samples. The values of the correlation length reported by Meneux et al. (2006) are slightly smaller than the values calculated here for the ALHAMBRA survey.

Quiescent galaxies show stronger clustering than late-type star-forming galaxies. Their correlation function parameters at large scales are nearly compatible within the errors with fixed values around $r_{0}=5 h^{-1} \mathrm{Mpc}$ and $\gamma=2$, but the clustering length is smaller than the one calculated at low redshift by Madgwick et al. (2003) for passive galaxies in the 2dFGRS and by Zehavi et al. (2011) for the red galaxies in SDSS. Instead the values of the amplitude of the correlation function reported by 
Skibba et al. (2014) at $z \sim 0.4$ for PRIMUS, by Coil et al. (2008) at $z \sim 0.9$ for the DEEP2 and by Meneux et al. (2006) agree with our results within the errors.

For both star-forming and passive galaxies the only discrepant measurement in Figure 8 is that corresponding to the PRIMUS samples at $z \sim 0.6$, which show values of $r_{0}$ significantly larger than those obtained by ALHAMBRA at similar redshifts (and also by DEEP2 at $z \sim 0.9$ ). This difference may be due to the fact that the PRIMUS survey includes the COSMOS field, which contains a large overdensity at this redshift affecting the clustering measurements (see the discussion in Section 2).

This segregation is generally explained by the tendency of red quiescent or early-type galaxies to form in dense environments while blue star-forming or late-type galaxies typically form in the field or in low-mass haloes (Dressler 1980; Goto et al. 2003; Bell et al. 2004; Thomas et al. 2005; Zucca et al. 2009; McNaught-Roberts et al. 2014).

\subsection{Dependence of the Bias on Spectral Type and Redshift}

To disentangle the evolution of the galaxy clustering of different populations from the overall growth of structure, we study the bias $b$ of our samples based on the projected correlation function measurements. We use a simple linear model with a constant and scale-independent bias. In this model the galaxy projected correlation function is given by

$$
w_{p}\left(r_{p}\right)=b^{2} w_{p}^{m}\left(r_{p}\right),
$$

where $b$ is the bias and $w_{p}^{m}\left(r_{p}\right)$ is the theoretical prediction for the projected correlation function of the matter distribution. Our model for $w_{p}^{m}$ is based on $\Lambda \mathrm{CDM}$ with cosmological parameters consistent with the WMAP7 results (Komatsu et al. 2011), including a normalization of the power spectrum $\sigma_{8}=0.816$. The matter power spectrum at the median redshift of each sample is obtained using the CAMB software (Lewis et al. 2000), including the non-linear HALOFIT corrections (Smith et al. 2003). We obtain the real-space correlation function $\xi(r)$ by a Fourier transform of the matter power spectrum and the final projected correlation function $w_{p}$ using Equation (3).

We fit this model to our data in the range $1.0<r_{p}<10.0 h^{-1} \mathrm{Mpc}$, corresponding mainly to the twohalo term of the correlation function. The best-fit value and uncertainty of the bias is obtained by the same method as described in Section 4.1 for the parameters of the power-law model. The results of these fits for each of our samples are listed in Table 2.

We show the evolution of the bias as a function of redshift for our different populations in the top panel of Figure 9 (red and blue squares). As expected we also see the effect of spectral segregation in this case, as the bias of early-type quiescent galaxies is consistently larger than that of late-type star-forming galaxies. The bias observed for the full population (not shown) is similar to that of the star-forming galaxies. For comparison we show the bias for dark matter haloes of a fixed mass (solid lines) according to the model of Tinker et al. (2005) and the values obtained by previous works for samples at

\footnotetext{
${ }^{24}$ In the case of Madgwick et al. (2003), as the bias values are not given explicitly, we derived the bias using their power law best fit at a scale of $5 h^{-1} \mathrm{Mpc}$.
}

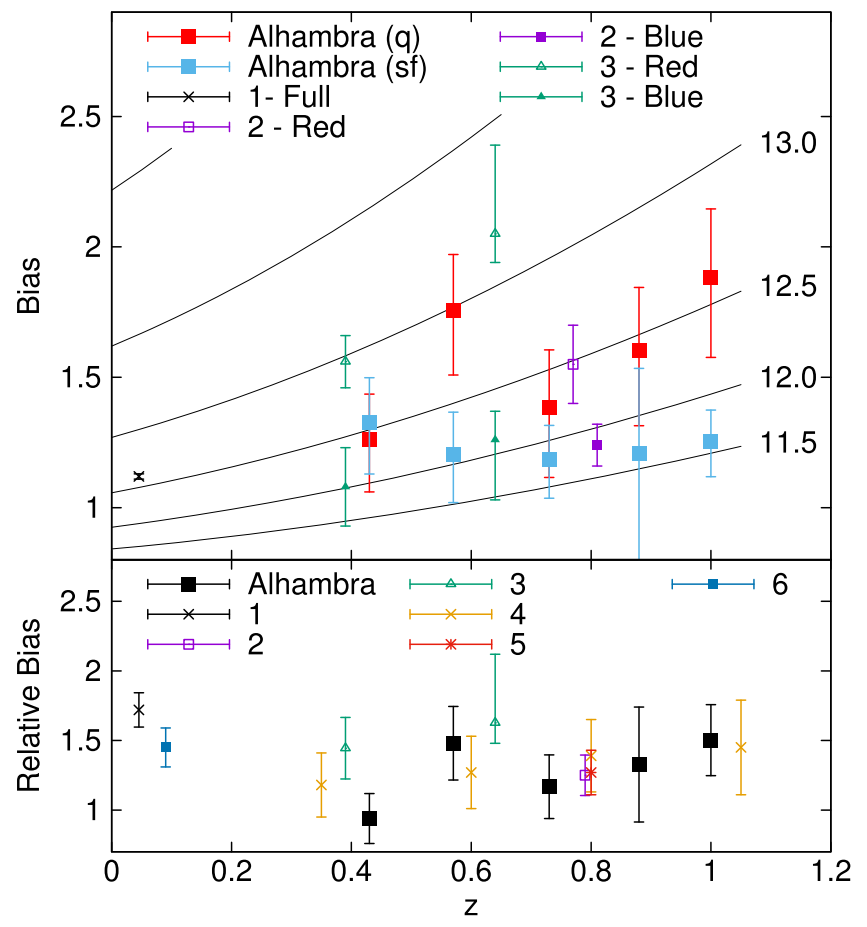

Figure 9. Galaxy bias of our quiescent (q) and star-forming (sf) galaxies as a function of their median redshift. Bias is estimated by a fit to Equation (14) as described in Section 4.2. The solid lines correspond to the bias of haloes of a fixed mass, according to the model of Tinker et al. (2005). These lines are labelled with the corresponding halo mass in terms of $\log _{10}\left[M_{\mathrm{h}} /\left(h^{-1} M_{\odot}\right)\right]$. For comparison we plot the results obtained by (1) Zehavi et al. (2011), (2) Coil et al. (2008), (3) Skibba et al. (2014), (4) Madgwick et al. (2003), (5) Marulli et al. (2013), (6) Meneux et al. (2006), and (7) de la Torre et al. (2011). Bias values from different authors have been adapted for the assumed cosmological parameters used in this paper.

similar redshift ranges and number densities. The bias values in those cases were obtained by a similar method as here and using compatible scale ranges. ${ }^{24}$

For the star-forming galaxies we obtain that their bias is approximately constant, with values $b \simeq 1.25$, over the range we explore. This explains the evolution of $r_{0}$ at large scales observed in Figure 8. If the bias is constant the main driver for the evolution of the galaxy clustering amplitude is the growth factor, therefore $r_{0}$ grows with cosmic time as observed. Given the uncertainties and the relatively slow evolution of the halo bias, the measured bias in this case is also consistent with the evolution of the bias of haloes with mass in the range $M_{\mathrm{h}} \simeq 10^{11.5}-10^{12} h^{-1} M_{\odot}$. As shown in Figure 9 our results for the star-forming population are fully consistent with those obtained for similar populations of blue galaxies in the the DEEP2 (Coil et al. 2008) and PRIMUS (Skibba et al. 2014) surveys.

The bias of quiescent galaxies shows a clear evolution, increasing with redshift, that is remarkably similar to the expected evolution of the bias for haloes of mass $M_{\mathrm{h}} \simeq 10^{12.5} h^{-1} M_{\odot}$. This clear evolution of the bias in this case compensates the clustering evolution due to the growth factor, resulting in an approximately constant value of $r_{0}$, as shown above in Figure 8. Our results for this population are consistent with the observed bias for red galaxies in the DEEP2 and PRIMUS surveys. We note that the bias measurement for the PRIMUS sample at $z \simeq 0.6$ may be affected by the presence of a large overdensity in the COSMOS field as noted above. 
The larger number of bins in redshift used in this ALHAMBRA analysis allows us to see more clearly this evolutionary trend.

In the bottom panel of Figure 9 we show the relative bias, defined as the ratio of the bias of quiescent galaxies over the bias of the star-forming ones, as a function of redshift. We also show for comparison the results from previous surveys at similar redshifts including, in addition to those shown in the top panel, the VVDS survey (Meneux et al. 2006) and the zCOSMOS-Bright survey (de la Torre et al. 2011). Meneux et al. (2006) used flux-limited samples with evolving galaxy density so their absolute bias measurements are not comparable to ours. The analysis by de la Torre et al. (2011) only provided values of the relative bias of their samples. At $z \sim 0$ we show the results from the 2dFGRS survey (Madgwick et al. 2003) and the SDSS (Zehavi et al. 2011). We note that in the two low redshift cases, the relative bias is calculated using a slightly different method: Madgwick et al. (2003) calculate it using the ratio of the galaxy variances $\sigma_{8 \text {,gal }}$ of the samples, while we have calculated the relative bias for SDSS as the ratio of the best-fit power laws of the two samples at a scale $r=5 h^{-1} \mathrm{Mpc}$ (see, e.g., Equation (9) of Norberg et al. 2002).

We obtain values of the relative bias in the range $b_{\text {rel }} \simeq 1-1.5$, consistent with all previous results at similar redshifts. The relative bias shows a very faint evolution slightly increasing with redshift. However, given the errors our results are also consistent with being constant. A similar faint trend is also seen for the VVDS results of Meneux et al. (2006). However, if we include the $z \sim 0$ values this evolution is broken and the best description of the results is a constant relative bias with redshift.

Overall, our results indicate that for samples selected by the same $B$-band luminosity and redshift, passive galaxies reside in haloes up to 10 times more massive than those hosting active galaxies. When studying the evolution with redshift, the observed bias suggests that quiescent galaxies (following a constant number density selection) reside in haloes of constant mass while this is not clear in the case of star-forming galaxies. In the latter case there seems to be an indication that they populate slightly more massive haloes at lower redshift. We will study the relation between galaxies and dark matter haloes using a more detailed HOD modeling in a future work.

\section{CONCLUSIONS}

The ALHAMBRA survey allows us to perform accurate clustering calculations with different segregation criteria. Its 23-filter photometry provides reliable galaxy parameters with good completeness out to very high redshifts $(z \sim 1.10)$, opening the possibility to analyze the galaxy clustering of different galaxy populations. In AM14 the authors chose to select galaxy samples using different luminosity thresholds while in this work we made a selection by spectral type. This selection follows the spectral classification of the ALHAMBRA photometric templates and has been proved to match remarkably well the usual selection by broadband color.

A rise of the correlation function at small scales is found as already noticed by Coil et al. (2008). We have been able to show that this trend holds at smaller scales and to characterize its redshift evolution.

Our sample allows us to measure the clustering properties of galaxy populations segregated by spectral type and their redshift evolution in a homogeneous way. At scales larger than $0.2 h^{-1} \mathrm{Mpc}$, quiescent galaxies cluster with a higher amplitude than star-forming ones. The difference is significant at the $2 \sigma$ level. There is also a significant hint of evolution $(2 \sigma)$ in the clustering amplitude of active galaxies while the clustering of the passive ones remains constant. These results are compatible with previous works in the literature, but in the present work we have increased the redshift resolution.

Regarding the small scales $\left(r_{p}<0.2 h^{-1} \mathrm{Mpc}\right)$ we find almost no change in the correlation function compared with the large scales in the quiescent population. On the other hand, the star-forming galaxies show a clear variation in the slope between small and large scales, which is possibly decreasing toward low redshifts.

Our measurements of the bias value for the different populations show strong segregation between them. The bias of the quiescent population clearly evolves with redshift following the expected behavior for haloes of approximate mass $10^{12.5} h^{-1} M_{\odot}$. The star-forming population bias remains basically constant over our observed redshift range, but it can still be compatible with the theoretical evolution of lower mass haloes $\left(M_{h} \sim 10^{11.5-12} h^{-1} M_{\odot}\right)$. As a consequence, the relative bias hints at a slow evolution, which would not be completely consistent with observations at $z \sim 0$.

We would like to thank an anonymous referee for comments that have improved the quality and readability of this paper. This work is based on observations collected at the GermanSpanish Astronomical Center at Calar Alto, which is jointly operated by the Max-Planck-Institut für Astronomie (MPIA) and the Instituto de Astrofísica de Andalucía (CSIC). This work was mainly supported by the Spanish Ministry for Economy and Competitiveness and FEDER funds through grants AYA2010-22111-C03-02 and AYA2013-48623-C2-2, and by the Generalitat Valenciana through project PrometeoII 2014/060. We also acknowledge support from the Spanish Ministry for Economy and Competitiveness and FEDER funds through grants AYA2012-39620, AYA2013-40611-P, AYA2013-42227-P, AYA2013-43188-P, AYA2013-48623C2-1, ESP2013-48274, AYA2014-58861-C3-1, Junta de Andalucía grants TIC114, JA2828, P10-FQM-6444, and Generalitat de Catalunya project SGR-1398. Begoña Ascaso acknowledge funding from the European Union's Horizon 2020 research and innovation program under the Marie Sklodowska-Curie grant agreement No. 656354.

\section{REFERENCES}

Abbas, U., \& Sheth, R. K. 2006, MNRAS, 372, 1749

Arnalte-Mur, P., Fernández-Soto, A., Martínez, V. J., et al. 2009, MNRAS, 394, 1631

Arnalte-Mur, P., Martínez, V. J., Norberg, P., et al. 2014, MNRAS, 441, 1783 Ascaso, B., Benítez, N., Fernández-Soto, A., et al. 2015, MNRAS, 452, 549 Bell, E. F., Wolf, C., Meisenheimer, K., et al. 2004, ApJ, 608, 752 Benítez, N. 2000, ApJ, 536, 571

Coil, A. L., Newman, J. A., Cooper, M. C., et al. 2006, ApJ, 644, 671 Coil, A. L., Newman, J. A., Croton, D., et al. 2008, ApJ, 672, 153

Cucciati, O., Iovino, A., Marinoni, C., et al. 2006, A\&A, 458, 39

Davis, M., \& Geller, M. J. 1976, ApJ, 208, 13

Davis, M., Meiksin, A., Strauss, M. A., da Costa, L. N., \& Yahil, A. 1988, ApJL, 333, L9

Davis, M., \& Peebles, P. J. E. 1983, ApJ, 267, 465

de la Torre, S., Guzzo, L., Kovač, K., et al. 2010, MNRAS, 409, 867

de la Torre, S., Le Fèvre, O., Porciani, C., et al. 2011, MNRAS, 412, 825

Domínguez-Tenreiro, R., \& Martínez, V. J. 1989, ApJL, 339, L9

Dressler, A. 1980, ApJ, 236, 351

Einasto, M. 1991, MNRAS, 252, 261

Giovanelli, R., Haynes, M. P., \& Chincarini, G. L. 1986, ApJ, 300, 77 
Goto, T., Yamauchi, C., Fujita, Y., et al. 2003, MNRAS, 346, 601

Guo, H., Zehavi, I., \& Zheng, Z. 2012, ApJ, 756, 127

Guo, H., Zehavi, I., Zheng, Z., et al. 2013, ApJ, 767, 122

Guzzo, L. \& The Vipers Team 2013, Msngr, 151, 41

Guzzo, L., Cassata, P., Finoguenov, A., et al. 2007, ApJS, 172, 254

Guzzo, L., Strauss, M. A., Fisher, K. B., Giovanelli, R., \& Haynes, M. P. 1997, ApJ, 489, 37

Hamilton, A. J. S. 1988, ApJL, 331, L59

Hamilton, A. J. S., \& Tegmark, M. 2004, MNRAS, 349, 115

Hartley, W. G., Almaini, O., Cirasuolo, M., et al. 2010, MNRAS, 407, 1212

Komatsu, E., Smith, K. M., Dunkley, J., et al. 2011, ApJS, 192, 18

Landy, S. D., \& Szalay, A. S. 1993, ApJ, 412, 64

Lewis, A., Challinor, A., \& Lasenby, A. 2000, ApJ, 538, 473

Li, C., Kauffmann, G., Jing, Y. P., et al. 2006, MNRAS, 368, 21

Loveday, J., Maddox, S. J., Efstathiou, G., \& Peterson, B. A. 1995, ApJ, 442, 457

Madgwick, D. S., Hawkins, E., Lahav, O., et al. 2003, MNRAS, 344, 847

Martínez, V. J., Arnalte-Mur, P., \& Stoyan, D. 2010, A\&A, 513, A22

Martínez, V. J., \& Saar, E. 2002, Statistics of the Galaxy Distribution (London: Chapman and Hall)

Marulli, F., Bolzonella, M., Branchini, E., et al. 2013, A\&A, 557, A17

Matthews, D. J., \& Newman, J. A. 2012, ApJ, 745, 180

McCracken, H. J., Peacock, J. A., Guzzo, L., et al. 2007, ApJS, 172, 314

McCracken, H. J., Wolk, M., Colombi, S., et al. 2015, MNRAS, 449, 901

McNaught-Roberts, T., Norberg, P., Baugh, C., et al. 2014, MNRAS, 445, 2125

Meneux, B., Le Fèvre, O., Guzzo, L., et al. 2006, A\&A, 452, 387
Moles, M., Benítez, N., Aguerri, J. A. L., et al. 2008, AJ, 136, 1325

Molino, A., Benítez, N., Moles, M., et al. 2014, MNRAS, 441, 2891

Norberg, P., Baugh, C. M., Gaztañaga, E., \& Croton, D. J. 2009, MNRAS, 396, 19

Norberg, P., Baugh, C. M., Hawkins, E., et al. 2002, MNRAS, 332, 827

Peebles, P. J. E. 1980, The Large-scale Structure of the Universe (Princeton, NJ: Princeton Univ. Press)

Phleps, S., Peacock, J. A., Meisenheimer, K., \& Wolf, C. 2006, A\&A, 457, 145

Pović, M., Huertas-Company, M., Aguerri, J. A. L., et al. 2013, MNRAS, 435, 3444

Roche, N., Eales, S. A., Hippelein, H., \& Willott, C. J. 1999, MNRAS, 306, 538

Scoville, N., Aussel, H., Benson, A., et al. 2007a, ApJS, 172, 150

Scoville, N., Aussel, H., Brusa, M., et al. 2007b, ApJS, 172, 1

Seljak, U. 2000, MNRAS, 318, 203

Skibba, R. A., Smith, M. S. M., Coil, A. L., et al. 2014, ApJ, 784, 128

Smith, R. E., Peacock, J. A., Jenkins, A., et al. 2003, MNRAS, 341, 1311

Stefanon, M. 2011, PhD thesis, Univ. de Valencia

Swanson, M. E. C., Tegmark, M., Hamilton, A. J. S., \& Hill, J. C. 2008, MNRAS, 387, 1391

Thomas, D., Maraston, C., Bender, R., \& Mendes de Oliveira, C. 2005, ApJ, 621,673

Tinker, J. L., Weinberg, D. H., Zheng, Z., \& Zehavi, I. 2005, ApJ, 631, 41

Zehavi, I., Weinberg, D. H., Zheng, Z., et al. 2004, ApJ, 608, 16

Zehavi, I., Zheng, Z., Weinberg, D. H., et al. 2011, ApJ, 736, 59

Zucca, E., Bardelli, S., Bolzonella, M., et al. 2009, A\&A, 508, 1217 